\title{
Thermally Activated Second-Order Recombination Hints toward Indirect Recombination in Fully Inorganic $\mathrm{CsPbl}_{3}$ Perovskites
}

Eline M. Hutter*i) and Tom J. Savenije*0

Department of Chemical Engineering, Delft University of Technology, van der Maasweg 9, $2629 \mathrm{HZ}$ Delft, The Netherlands

\section{Supporting Information}
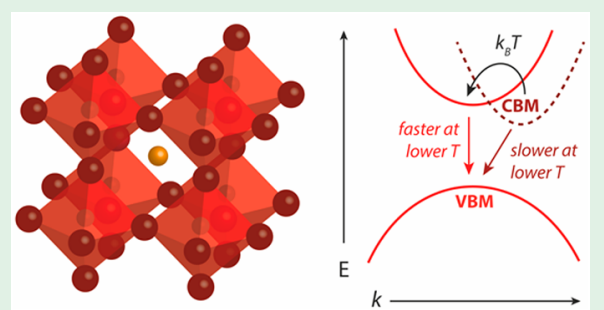

ABSTRACT: The relationship between the dipole moment of the methylammonium cation and the optoelectronic properties of lead halide perovskites remains under debate. We show that both the temperature-dependent charge carrier mobility and recombination kinetics are identical for methylammonium and cesium lead iodide, indicating that the role of the monovalent cation is subordinate to the lead iodide framework. From the observation that for both perovskites the electron-hole recombination is thermally activated, we speculate that the bandgap is slightly indirect.

\section{A} pplication of lead halide perovskites with the general structure $\mathrm{ABX}_{3}$ in solar cell devices yields efficiencies close to $23 \%{ }^{1}$ This is attributed to the combination of high absorption coefficients, sufficiently high charge carrier mobilities, and long lifetimes, enabling efficient collection of excess charges. Previous work on methylammonium lead iodide or bromide $\left(\mathrm{MAPbX}_{3}\right)$ has shown that these long lifetimes could be related to the presence of the Rashba effect, resulting in a slightly indirect bandgap. ${ }^{2-8}$ Importantly, optical excitation of charges still predominantly occurs via direct transitions, while recombination involves an indirect transition. However, it has remained under debate whether these effects are related to the dipole moment of the organic cation.

In this work, we show that both the temperature-dependent mobility and recombination kinetics in $\mathrm{CsPbI}_{3}$ follow the same trend as in $\mathrm{MAPbI}_{3}$. Importantly, in both cases, the second-order recombination of free charges is thermally activated, consistent with an indirect recombination pathway.
The $\mathrm{CsPbI}_{3}$ films are prepared using physical vapor deposition, yielding metastable orthorhombic black-phase $\mathrm{CsPbI}_{3} \cdot{ }^{9-11}$ Figure 1 shows intensity-normalized TimeResolved Microwave Conductivity (TRMC) traces recorded at 293,260 , and $220 \mathrm{~K}$, for charge carrier densities in the regime
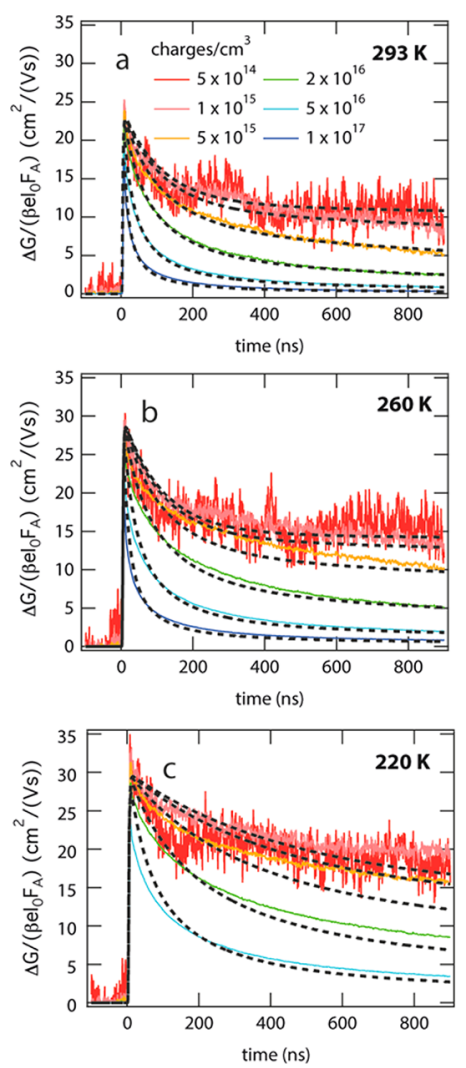

Figure 1. TRMC traces for vapor-deposited black-phase $\mathrm{CsPb}_{3}$, recorded at $293 \mathrm{~K}(\mathrm{a}), 260 \mathrm{~K}(\mathrm{~b})$, and $220 \mathrm{~K}$ (c) for initial charge carrier densities of $5 \times 10^{14}$ to $10^{17} \mathrm{~cm}^{-3}$. The modeled traces are added as black dashed lines.

Received: July 1, 2018

Accepted: July 18, 2018

Published: July 18, 2018 


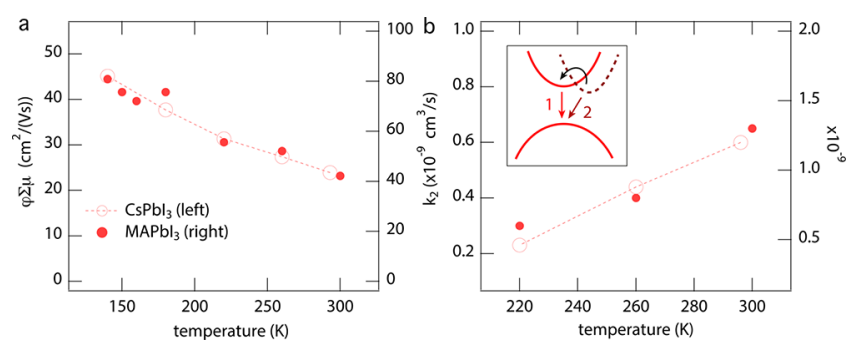

Figure 2. Effective mobility $\varphi \sum \mu$ (a) and $k_{2}$ (b) as a function of temperature for $\mathrm{CsPbI}_{3}$ (open circles, left axis) and $\mathrm{MAPbI}_{3}$ (closed circles, right axis, data from ref 7 ). The dotted lines between the open circles are added to guide the eye.

between $10^{15}$ and $10^{17} \mathrm{~cm}^{-3}$. From the maximum signal height, we deduce that the room-temperature mobility is around 25 $\mathrm{cm}^{2} /(\mathrm{V} \mathrm{s}) .{ }^{10}$ Interestingly, on decreasing the temperature, the mobility in $\mathrm{CsPbI}_{3}$ gradually increases, following a temperaturedependent trend similar to that observed for $\mathrm{MAPbI}_{3}$ (see Figure 2a) given by $\mu \propto T^{-1.5}$.,12 This shows that the charge carrier mobility in lead iodide perovskites is mainly limited by phonon scattering, ${ }^{7}$ independent of the monovalent cation. Remarkably, not only the mobility but also the lifetime of mobile charges follows the same temperature dependence as that of $\mathrm{MAPbI}_{3}$. That is, for a range of excitation densities between $5 \times$ $10^{15}$ and $10^{17} \mathrm{~cm}^{-3}$, the lifetime is enhanced on lowering the temperature. Because this corresponds to a regime in which the lifetime decreases with increasing initial charge carrier densities, we can conclude that higher-order recombination is retarded with decreasing temperatures. Below $5 \times 10^{15} \mathrm{~cm}^{-3}$, the lifetime is not dependent on the excitation density, indicating a firstorder process such as trap-assisted recombination.

To quantify this, we fitted the experimental data at $220 \mathrm{~K}<T$ $<300 \mathrm{~K}$ using our previously described kinetic model (Figure $\mathrm{S} 2),{ }^{13}$ as shown by the dashed lines in Figure 1. The kinetic parameters are listed in Table S1. In Figure $2 b$, the second-order recombination rate $\left(k_{2}\right)$ is plotted for $\mathrm{CsPbI}_{3}$ with values previously found for $\mathrm{MAPbI}_{3}{ }^{7}$ Importantly, although the absolute values are different, in both cases the $k_{2}$ at $220 \mathrm{~K}$ is less than half its room-temperature value. This is in contrast with the temperature-dependent trend in recombination rate typically found in direct semiconductors and previously reported for $\mathrm{MAPbI}_{3}$ at high fluence $\left(>10^{17} \mathrm{~cm}^{-3}\right),{ }^{12,14,15}$ which is several orders of magnitude higher than the charge densities presented here. Although these previous reports ${ }^{12,15}$ suggest that direct recombination dominates at higher charge densities, our results show that in both $\mathrm{CsPbI}_{3}$ and $\mathrm{MAPbI}_{3}$, second-order recombination is actually a thermally activated process for charge densities ranging from $10^{15}$ to $10^{17} \mathrm{~cm}^{-3}$. Previously, we hypothesized that this was due to the conduction band minimum being slightly shifted in $k$-space from the valence band maximum, ${ }^{7}$ resulting in an indirect bandgap from which recombination is momentum-forbidden. In addition, the high absorption coefficients ${ }^{10}$ indicate the presence of a direct transition. The origin of the thermally enhanced recombination rates may therefore be twofold: (1) thermal energy releases electrons from the CBM to a state from which direct recombination is possible and (2) the electrons decay from the CBM to the VBM via indirect recombination on interacting with a phonon, see inset in Figure $2 \mathrm{~b}$. The present observations suggest that the indirect recombination pathway is not related to (collective) orientations of methylammonium dipoles.
We note that the increased lifetime observed upon lowering the temperature is not related to reabsorption of emitted photons. That is, the external photoluminescence quantum efficiency (PLQE) in $\mathrm{CsPbI}_{3}$ is far below $1 \% .{ }^{10}$ Even in the case of very poor output coupling and a 10-fold increase in PLQE on lowering the temperature (see Figure S1), still more than $95 \%$ of the second-order recombination is nonradiative and therefore cannot be reabsorbed. Additional scenarios to explain the thermally enhanced recombination include charge immobilization into shallow traps or the formation of large polarons. However, because both of these do not satisfactorily explain the experimental temperature-dependent mobility following $T^{-1.5}$, , 16 these are in our opinion unlikely to dominate the charge carrier recombination properties. Instead, the combination of increasing mobility and retarded second-order recombination on decreasing the temperature suggests an indirect recombination pathway for mobile charges. The low PLQE indicates that the second-order recombination between mobile charges is mainly nonradiative, which could mean that indirect recombination does not result in the emission of a photon. Most importantly, our present results show that both the temperature-dependent mobility and recombination mechanism are fully dominated by the lead iodide framework instead of the dipole moment of the organic cation.

\section{ASSOCIATED CONTENT}

\section{Supporting Information}

The Supporting Information is available free of charge on the ACS Publications website at DOI: 10.1021/acsenergylett.8b01106.

Additional figures and experimental methods (PDF)

\section{AUTHOR INFORMATION}

\section{Corresponding Authors}

*E-mail: E.M.Hutter@tudelft.nl.

*E-mail: T.J.Savenije@tudelft.nl.

ORCID $\odot$

Eline M. Hutter: 0000-0002-5537-6545

Tom J. Savenije: 0000-0003-1435-9885

Notes

The authors declare no competing financial interest.

\section{ACKNOWLEDGMENTS}

Sanjana Chandrashekar is acknowledged for sample preparation.

\section{REFERENCES}

(1) http://www.nrel.gov.

(2) Motta, C.; et al. Nat. Commun. 2015, 6, 7026.

(3) Etienne, T.; et al. J. Phys. Chem. Lett. 2016, 7, 1638-1645.

(4) Azarhoosh, P.; et al. APL Mater. 2016, 4, 091501.

(5) Niesner, D.; et al. Phys. Rev. Lett. 2016, 117, 126401.

(6) Wang, T.; et al. Energy Environ. Sci. 2017, 10, 509-515.

(7) Hutter, E. M.; et al. Nat. Mater. 2017, 16, 115-120.

(8) McKechnie, S.; et al. 2017, arXiv:1711.00533v1 [cond-mat.mtrlsci].

(9) Frolova, L. A.; et al. J. Phys. Chem. Lett. 2017, 8 (1), 67-72.

(10) Hutter, E. M.; et al. ACS Energy Lett. 2017, 2, 1901-1908.

(11) Sutton, R. J.; et al. ACS Energy Lett. 2018, 3, 1787-1794.

(12) Milot, R. L.; et al. Adv. Funct. Mater. 2015, 25 (39), 6218-6227.

(13) Hutter, E. M.; et al. J. Phys. Chem. Lett. 2015, 6, 3082-3090.

(14) 't Hooft, G. W.; et al. Appl. Phys. Lett. 1983, 42 (9), 813-815.

(15) Davies, C. L.; et al. Nat. Commun. 2018, 9 (1), 1-9.

(16) Sendner, M.; et al. Mater. Horiz. 2016, 3 (6), 613-620. 